\title{
Leitlinien der FMH zur Anerkennung von Fort- bildungsveranstaltungen im Rahmen der FBO
}

1. Inhalt und Präsentation von Fortbildungsveranstaltungen sollen einzig durch Ärztinnen und Ärzte bzw. ärztliche Fachgremien bestimmt werden.

2. Das gleiche gilt für die Bestimmung von Ort und Zeitpunkt der Durchführung einer Fortbildungsveranstaltung.

3. Nur die veranstaltenden Ärztinnen und Ärzte bzw. die ärztlichen Fachgremien können bei den von der Fortbildungsveranstaltung betroffenen Fachgesellschaften die Anerkennung beantragen.

4. Die Möglichkeiten der Prävention, Diagnose und Therapie sollen vollständig, nach den Regeln der evidenzbasierten Medizin (EBM) und unter Berücksichtigung ihrer Wirtschaftlichkeit dargestellt werden.

5. Stehen für die besprochene Diagnose oder Therapie mehrere wirksame Arzneimittel,
Medizinprodukte oder Verfahren zur Verfügung, ist ein umfassender Vergleich anzustreben.

6. Finanzielle Mittel aus Sponsoring sind für die Organisation der Fortbildungsveranstaltung, Honorierung der Referenten und deren Spesen zu verwenden.

7. Allfällige kommerzielle Interessen der Referenten, finanzielle Verbindungen zum Sponsor, Beratertätigkeit im Auftrag des Sponsors oder Forschungsunterstützung durch den Sponsor müssen die Referenten und Organisatoren der betreffenden Fortbildungsveranstaltung offenlegen und im Programm ankündigen.

Diese Leitlinien wurden vom Zentralvorstand der FMH an seiner Sitzung vom 21. November 2003 genehmigt.

\section{Directives de la FMH pour la reconnaissance de sessions de formation continue dans le cadre de la RFC}

1. Le contenu et la présentation des sessions de formation continue doivent être déterminés uniquement par des médecins ou par des commissions médicales spécialisées.

2. Le même principe s'applique à la détermination du lieu et de la date de la session de formation continue.

3. Seuls les médecins ou les commissions médicales qui organisent les sessions de formation continue peuvent déposer une demande de reconnaissance de ces dernières auprès de la société de discipline médicale concernée.

4. Les possibilités existantes en matière de prévention, de diagnostic et de traitement doivent être présentées dans leur intégralité, selon les principes de la médecine basée sur des donnés probantes (EBM) et en tenant compte du critère d'économicité.

5. Si, pour le diagnostic ou la thérapie prévue, plusieurs médicaments, produits thérapeutiques ou procédés efficaces sont à disposi- tion, il convient d'effectuer une comparaison approfondie.

6. Les moyens financiers mis à disposition par les commanditaires sont à utiliser exclusivement pour l'organisation des sessions de formation continue, pour le versement des honoraires des intervenants et le remboursement des frais de ces derniers.

7. Les éventuels intérêts commerciaux des intervenants, les liens financiers avec les commanditaires, les activités de conseil effectuées à la demande de ces derniers ou le soutien qu'ils accordent à des travaux de recherche doivent être mentionnés ouvertement par les intervenants et les organisateurs, et inscrits dans le programme de la session de formation continue correspondante.

Ces directives ont été approuvés par le Comité central de la FMH lors de sa séance du 21 novembre 2003. 